\title{
Thrust Control, Stabilization and Energetics of a Quadruped Running Robot
}

\author{
Joaquin Estremera ${ }^{1}$ and Kenneth J. Waldron ${ }^{2} 3$ \\ ${ }^{1}$ Industrial Automation Institute -CSIC, Arganda del Rey, Madrid, Spain \\ ${ }^{2}$ Stanford University, Department of Mechanical Engineering, Palo Alto, CA, USA. \\ jestremera@iai.csic.es, kwaldron@stanford.edu
}

\begin{abstract}
In order to achieve powered autonomous running robots it is essential to develop efficient actuator systems, especially in the case of the actuatorsfor generating the radial thrust in the legs. In addition, the control of the radial thrust of the legs can be a simple, effective method for stabilizing the body pitch in a running gait. This paper presents the mechanical systems, the models and control strategies employed to generate and control leg thrust in the KOLT quadruped running robot. An analytical model of the electro-pneumatic leg thrusting system is presented and analyzed to evaluate its performance and to facilitate the design of control strategies. Several experiments have been conducted to estimate the energy losses and determine their origins as well as to compute the energetic efficiency of the actuation system. Two thrust control methods are also proposed and tested experimentally. The closed loop method regulates thrust through the control of the hip liftoff speed, a conceptually simple control strategy that has the property of-stabilizesing the body pitch in pronk and trot gaits without the need of central feedback, even on irregular terrain. An-The open loop control method that can-regulates the energy added in each hop based on the model of the actuator system-is also presented. The efficacy of these models and techniques is tested in several planar trot and pronk experiments, and the results obtained are presented and analyzed focusing particularly on the body stabilization, the power consumption, and the energetic efficiency-of the locomotion.
\end{abstract}

\footnotetext{
${ }^{3}$ The authors would like to thank Paul Csonka and Surya P. N. Singh for their assistance in this work. The authors also acknowledge the support of the National Science Foundation, Grants Nos. IIS-0208664 and IIS-0535226, and the Secretaria de Estado de Universidades of the Spanish Ministry of Education and Science.
} 
Key words: Running robot, legged machine, quadruped, fast locomotion, dynamic stability, trot, pronk, gallop.

\section{Introduction}

Research on legged robots has been carried on for more than four decades. In this period multilegged, statically stable walking robots have reached an acceptable degree of functionality (Song and Waldron, 1989; Plustech Oy, 1995; Gonzalez de Santos et al., 2000; Cepolina et al., 2006) and have proved to be to be superior to conventional vehicles in certain aspects, such as terrain adaptability. However, the inherent slow speed of legged machines is one of the main drawbacks that have prevented their use in practical applications. Thus, an important challenge in the field of legged robotics is to conciliate the advantages of legged machines (mainly the ability to negotiate irregular terrain) and the possibility of reaching speeds similar to those observed in the fastest running animals.

However, However, in contrast with walking robots, only a reduced number of rumning legged machines have been reported to run successfully in the last two decades. This fact reveals that several major technical problems and scientific challenges must still be solved before high speed locomotion is achieved with a fully operational, power autonomous and self-contained legged robot.

One of the main challenges to be solved-is the design of mechanical systems able to deliver the energy needed to sustain high speed locomotion during the short and intermittent periods of foot-ground contact. When this constraint is considered jointly with acceptable energetic efficiency and weight, the demands placed on the actuation systems are difficult to fulfill. This applies especially to the actuators generating radial leg thrust (i.e. producing leg extension) which have to-handle higher loads. The systems described in (Brown and Zeglin, 1998; Ahmadi and Buehler, 1999; Waldron and Nichol, 2004) represent different attempts to achieve energy efficient locomotion systems. Ahmadi and Buehler (1999) presented a study of the energetics of the electrically actuated monopod running robot ARL-II, showing better efficiency thant any other previous legged robot. However, a detailed experimental energetic study of fast quadrupedal locomotion, describing accurately the efficiency and the contribution of the different loss sources is still missing to date. The study of the energetics of locomotion are-is considered essential by the 
authors in order to design power savingefficient actuation systemors to progress toward practical, pachieve power autonomous, fast legged robots.

The stabilization and control of fast dynamic gaits is another challenge that is yet to be solved. In animals, high speed gaits are supposed to be stabilized by the passive dynamics of the musculo-skeletal structure and by simple feed-forward mechanisms, more than by controllers relying on central feedback. For example,-Herr and McMahon (2000; 2001) hypothesized and proved in simulation that trot and gallop gaits could be stabilized without the need of feedback of the body position and orientation. Instead, animals would use a more local control loop to regulate the torque in the hip and shoulder joints of the supporting legs so that the resulting linear speed of the feet is slightly higher (for rear legs) or slightly lower (for fore legs) than the body target speed. That would produce a "shoulder braking" and "hip thrusting" effect that stabilizes the body pitch motion.

In the case of robots, two main approaches have been proposed to generate stable dynamic gaits. The first approach consists of obtaining the return maps that describe the dynamic behavior of the machine to find regions in which the gait is passively stable. Murphy_(Raibert, 1986) discovered that the passive stability of a bound gait could be predicted as a function of the non-dimensional moment of inertia-(Raibert, 1986), defined by:

$$
\hat{I}=I /\left(m l^{2}\right)
$$

where $I$ is the moment of inertia of the robot around its pitch axis, $m$ is its mass and $l$ is half the distance between fore and rear hips. The conditionbound gait is passively stable only if $\hat{I}<1$; ensures that the bound gait is passively stable, anda condition that_-sets a design constraint for bounding robots. For example,_-in Raibert's quadruped (Raibert, 1990) $\hat{I}$ was estimated to be 0.422 (Neishtadt and $\mathrm{Li}, 1991$ ), so a stable bounding gait could be obtained without the need of-any active control. Berkemeier (1998) derived approximate analytic return maps for the pronk and the bound gaits on planar models running in place. He found that in a pronk gait-and for a given non-dimensional inertia, different discrete regions of stability existed depending on the apex height. Another example of this approach is the method used to find a stable cycle for the running robot SCOUT II (Poulakakis et al., 2003). A return map obtained by numerical simulation was employed to find the leg 
touchdown angles for the legs-that generated a passively stable bounding gait, which was successfully implemented in the robot.

A second strategy consists of actively controlling the attitude of the machine to stabilize the gait. In Raibert's quadruped, trot, pace and pronk gaits were stabilized by applying torques to the hip joints during leg stance (Raibert, 1990), which were computed as a function of the body pitch and roll angles and rates by a PD controller. In (Berkemeier, 1996) another high level controller was proposed to stabilize a pronk gait by independently regulating fore and rear leg thrust. Differential leg thrust was also used by Marehfka (2003) to stabilize bound and gallop gaits. The leg thrusts (and also the leg touchdown angleswere computed at the top of flight by a fuzzy controller tuned in simulation to achieve the desired state (body forward speed, height, attitude and angular rate) at the next top of flight. Although the last two methods proved to be effective in simulation they have not been tested in real quadrupeds.

The regulation of leg thrust as an effective technique for controlling and stabilizing real running quadruped robots has not been addressed sufficiently in the past. For example, Raibert's quadruped; did not use any method to actively regulate leg thrust; instead, the leg was extended a fixed length during leg stance. However, an alternative method for regulating the energy injected by the leg extension was also proposed (Raibert, 1986). In the SCOUT II quadruped robot, the leg longitudinal-radial thrust cannot be actuated independently, and its single actuator per leg adds energy to the bound gait by rotating the leg around its-hip joint-while a spring provides the required-compliance. The robot SCAMPER (Furusho et al., 1995) performed a bound gait by actuating its knee with an electric motor working in speed control mode, a method that is a precursor of that presented in section 4.14.14.1. However, to the authors' best knowledge, the repercussions of this technique in the stability of the bounding gait was not addressed.

Finally, the development of functional localization systemsensorss able to estimate the orientation and speed of the machine on irregular terrain in the particular working conditions characterized by running (repeated impacts, vibration and high accelerations) is yet another major problem to be solved. Given the difficulty of estimating the overall state of the machine, it is important to develop controllers that are robust against errors in the estimation or that even do not need this kind of feedback (Iida and Pfeifer, 2004). 
This paper presents ongoing work on the KOLT robot related to leg thrust control, body stabilization and energetics; of simple running gaits, namely the trot and the pronk gaits. This work is intended to be a step towards the implementation of complex high speed gaits such as the gallop. The paper is organized as follows: The experimental platform, the KOLT quadruped, is described in section 22z2. A simple control strategy to stabilize trot and pronk gaits through the regulation of leg radial thrust is proposed in section $\underline{33} 3$. Two methods implemented in the robot to control leg thrust and a model of the leg electromechanical system are described in section $\underline{444}$. Experimental and simulation results showing the performance of these methods, and evaluating operation of the actuator systems in pronk and trot gaits are presented and discussed in section_-0555. Finally, section $\underline{006} \underline{6}$ offers some conclusions.

\section{The KOLT quadruped platformDescription of the System}

The experimental platform employed is the KOLT (Nichol et al., 2004) quadruped (see Figure 1Figure 1Figure 1Figure 1 and Table 1Table 1Table 1Table 1). This robot measures $1.75 \times 0.60 \times 0.80 \mathrm{~m}$ and weights $80 \mathrm{Kg}$. The distance between hips is $0.73 \mathrm{~m}$, and the leg length is $0.65 \mathrm{~m}$ when the leg is extended. The-moment of inertia of the robot around the pitch (transversal) axis that passes through the center of masses is $19.1 \mathrm{Kg} \mathrm{m}^{2}$ when the legs

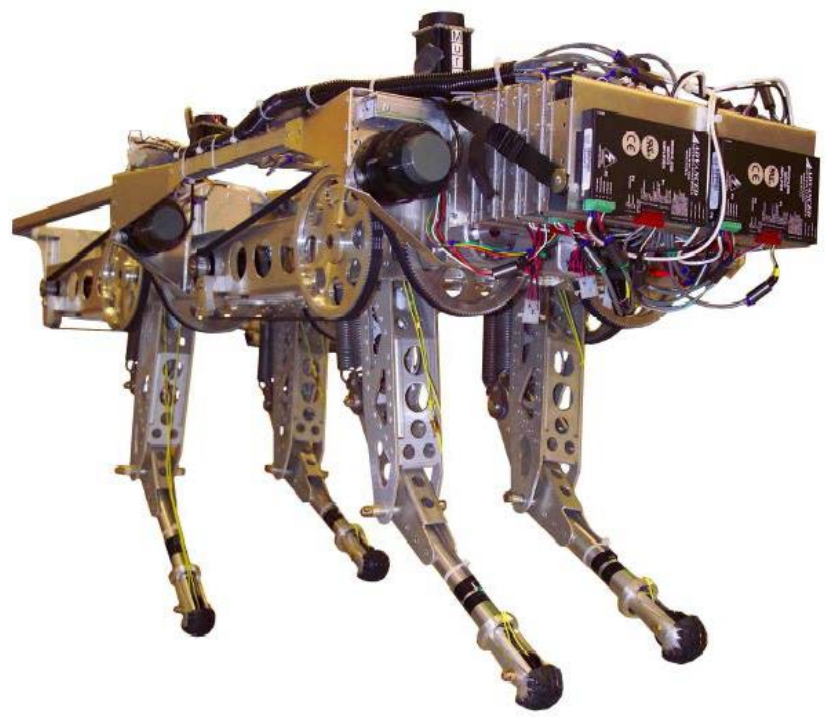

Figure 1. The KOLT quadruped robot. 
are in their neutral position. Hence, the non-dimensional moment of inertia-is $\hat{I}=1.77$, $\underline{\text { is }}$ well outside the region in which the bounding gait is passively stable.

The legs are based on a mammal type configuration, and they have three active degrees of freedom driven by brushless motors. The hip pitch and roll joints are driven by high torque motors for which the reduction ratio is only $6: 1$. Since the joint mechanical impedance is proportional to the square of the reduction ratio, this low ratio yields a very low joint impedance. In this way, ifWhen the electric motor is inactive ${ }_{2}$ the hip joints-can act approximately as free joints (despite of parasitic torques), -in-order to allowing for a natural dynamic behavior of the quadruped during leg stance. Thee knee electro-pneumatic actuation system has been designed to maximize the thrust and the energetic efficiency. The knee joint is actuated in an agonist antagonist wayby a brushless motor that produces leg flexion and a pneumatic spring which produces leg extension (see Figure 2Figure ZFigure 2Figure 2). The knee motor actuates the knee joint by means of a cable and an arrangement of pulleys that allow decoupling the knee and the thigh motionThe shaft of the knee motor directly drives a capstan on which a cable is wound. The arrangement of this eable around two pulleys located in the thigh joint and near the foot permits the decoupled acturtion of the knee joint without interfering in the thigh joint motion (Waldron and Nichol, 2004). The pneumatic spring provides the necessary leg compliance at landing and makes possible the storage of elastic energy. The stiffness of the resulting virtual leg depends on the knee angle (Schmiedeler, 2001), and is $15.9 \mathrm{kN} / \mathrm{m}$ when the leg is extended and the pneumatic spring is pre-charged at 100 p.s.i. Two additional elements, a-check valve and an air reservoir, permit storage of elastic energy during flight and control of the amount of energy injected to the system in the support phase. The $\underline{A n}$ air reservoir is connected to the pneumatic spring through a one-way check valve, which passively permits flow from the spring to the reservoir when the leg flexes (i.e., when the spring is compressed) and prevents flow in the opposite direction when the leg extends. This valve can be actively opened by the control system to allow air to flow back from the reservoir to the spring. During a hop the leg alternates through-The leg working cycle is composed of four phases-which are summarized next:

1. Phase 1: After the foot liftoff, the knee motor flexes the knee ${ }_{2}$ compressing the pneumatic spring, and pumping pressurized air into the reservoir to store energy-and 
to produce ground clearance. The hip motors move the foot to the desired touchdown position.

2. Phase 2: The knee motor unwinds the cable, letting the pneumatic spring extend the leg in preparation for landing. The ene-way-check valve retains the pressurized air in the reservoir. The hip motors maintain the foot in the desired touchdown angle.

3. Phase 3: After landing, the leg is-flexesed under the weight of the robot and, thus, the spring is compressed ${ }_{2}$. Kinematic and potential energy of the robot are stored in the spring as storing elastic energy. Hip motors are inactive during stance so the hip acts as a passive joint.

4. Phase 4: After reaching the maximum leg compression, the valve is opened so thatand the pressurized air stored in the reservoir ean-returns to the spring. The leg extends while the elastic energy stored in the spring and reservoir is converted into kinetic and potential energy causing the liftoff of the foot and beginning a new cycle.

The design has two main advantages: First-, the work done by the electric motors can be stored during the relatively long flight phase, and then released rapidly during the relatively short stance phase. This means that lower power motors can be employed. Second, the use of pneumatics provide additional means for controlling the energy stored and released by the spring, as explained in this paper.

Each leg is controlled by a $\mathrm{n}$ independent-microcontroller in charge of executing thethat
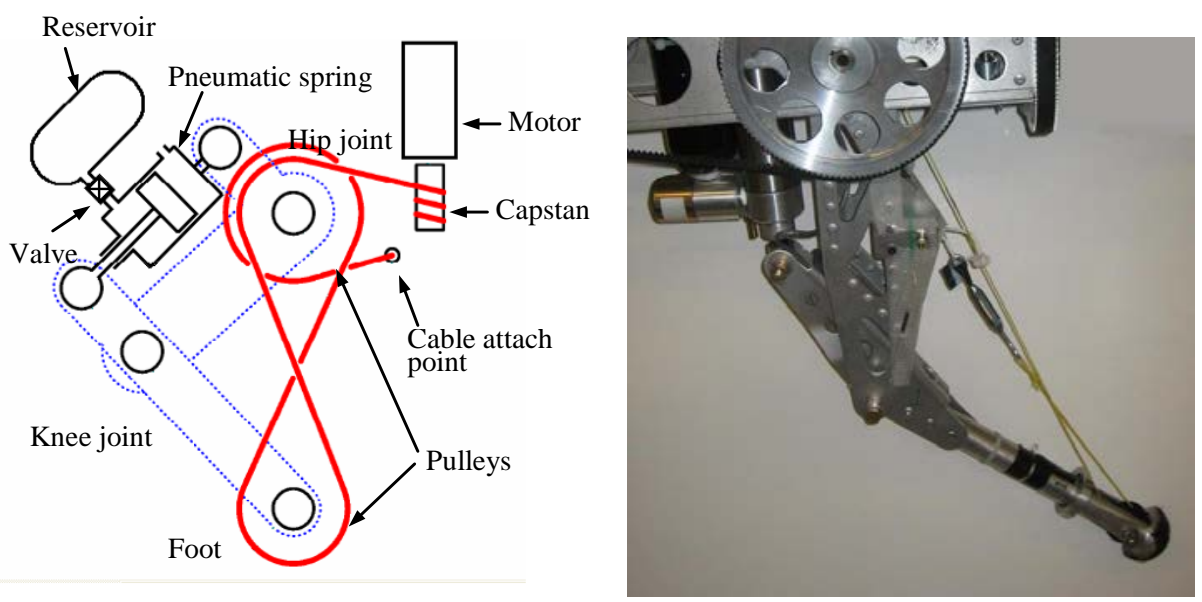

Figure 2. Schematic and picture of the electro-pneumatic leg thrusting system. 
controls low level joint positions eontrol as well asand generatesing the leg working cycle through the four states described above. Feedback is provided by a foot switch activated during ground contact and encoders in the motor-shaftss. Throughout the stance period the eable that transmits the motion from the knee motor to the shank is slack (as the knee motor and the knee joint do not move synchronously) and for this reason an additional encoder is placed on the knee.During stance, the cable joining the knee motor and the shank is slack, and the motor and the knee joint do not move synchronously. For this reason, an additional encoder is placed on the knee.

A central computer is in charge of coordinatesing the four leg microcontrollers and estimatesing the overall state of the robot. A localization system combining data from-an inertial measurement system (IMU) and infrared rangefinders aimed towards the ground is employed to determines the pitch and roll angles of the body as well asand its height-above the ground (Singh and Waldron, 2005). In the current development stageCurrently, the KOLT robot is supported by a boom that confines its motion to a plane: vertical and longitudinal motion and rotation about the pitch axis are allowed, while lateral motion and roll and yaw rotations are impeded. An encoder is mounted on the boom to measure the pitch angle accurately in order to tme the IMU/Rangefinder localization system. This encoder is employed as body control input in the experiments presented here. $\underline{\text { In the }}$ experimental results presented in this paper, the body pitch is measured accurately with an encoder mounted on this boom. However, these measurements are not employed as feedback for the controllers.

Each leg microcontroller estimates the forward speed of the body based on the thigh angular speed during stance. This information is sent to the main- central computer where combines these estimations-from the four legs are combined to obtain the overall body speed. The touchdown position of the feet is computed as a linear function of the error in forward speed (Raibert, 1990) and the error in position on the treadmill on which the robot runs (Brown and Zeglin, 1998; Ahmadi and Buehler, 1999):

$$
x_{f}=\left(v-v_{T}\right) T_{\text {support }} / 2+k_{V} v+k_{P}\left(x-x_{d}\right)
$$

where $x_{f}$ is the-_position of the foot referred to the hip, $v_{T}$ is the treadmill speed, $T_{\text {support }}$ is the support time of the fooeet, $\underline{x} \forall$ and $* \underline{v}$ are the body position and speed, $x_{d}$ is the desired 
Table 1. Characteristics of the KOLT quadruped robot.

\begin{tabular}{|l|l|l|}
\hline \multirow{4}{*}{ Robot } & Weight & $80 \mathrm{Kg}$ \\
\cline { 2 - 3 } & $\mathrm{I}_{\mathrm{yy}}$ & $19.1 \mathrm{Kgm}^{2}$ \\
\cline { 2 - 3 } & $\hat{\mathrm{I}}$ & 1.77 \\
\cline { 2 - 3 } & Length & $1.75 \mathrm{~m}$ \\
\cline { 2 - 3 } & Width & $0.60 \mathrm{~m}$ \\
\cline { 2 - 3 } & Height & $0.80 \mathrm{~m}$ \\
\hline Body & Weight & $36.8 \mathrm{Kg}$ \\
\cline { 2 - 3 } & $\mathrm{I}_{\mathrm{xx}}$ & $1.6 \mathrm{Kgm}^{2}$ \\
\cline { 2 - 3 } & $\mathrm{I}_{\mathrm{yy}}$ & $14.9 \mathrm{Kgm}^{2}$ \\
\cline { 2 - 3 } & $\mathrm{I}_{\mathrm{zz}}$ & $15.9 \mathrm{Kgm}^{2}$ \\
\cline { 2 - 3 } & Length & $1.75 \mathrm{~m}$ \\
\cline { 2 - 3 } & Width & $0.60 \mathrm{~m}$ \\
\cline { 2 - 3 } & Height & $0.19 \mathrm{~m}$ \\
\hline \multirow{5}{*}{ Leg } & Weight & $11.0 \mathrm{Kg}$ \\
\cline { 2 - 3 } & Thigh length & $0.35 \mathrm{~m}$ \\
\cline { 2 - 3 } & Shank length & $0.35 \mathrm{~m}$ \\
\hline
\end{tabular}

position of the body and $k_{V}$ and $k_{P}$ are experimentally tuned gains. The touchdown angle is referred to the vertical so- $\underline{\text { the }}$ attitude of the body and the touchdown position $x_{f}$ aremeasured by the attitude sensors was employed to determine the final final thigh angles.

Very simple controllers were-are employed to produce pronk and trot gaits. In the case of the-pronk, each leg works independently and no coordination mechanisms employed to synchronize the phases of the legs. In the ease of the trot gait, data from the foot contact switches are employed to alternate the states of the two pairs of legs: a pair of diagonal (non-adjacent) legs waits flexed (i.e., they remain-in phase 1) until the other pair has landed and taken off the ground. The hip joints doid not exert torques during stance in order to control the attitude nor to propel the body, acting thus as free joints. The leg radial thrust ${ }_{2}$ (i.e., the thrust-provided by the knee actuator system ${ }_{2}$ ) was-is controlled with different methods, as explained in the following sections.

\section{Liftoff Speed Control for Body Stabilization}

A simple strategy defined as the maximum linear speed of the hip related to the foot (in the foot-hip direction), is controlled in closed loop mode during the leg stance. In this way, the initial speed of the hip at the beginning of the flight phase can be controlled, and thus the body 
take-off speed and the body top of flight height can be regulated. This approach presents several advantages when compared with other thrust regulation methods:

1.Liftoff speed is easily measurable in a real robot, thanks to the joint position sensors,

Formatted: Bullets and Numbering while magnitudes such as the impulse or the energy added by the leg thrust should be estimated or measured using more complex sensors.

This simple low_-level controller can help to stabilize gaits in which the body remains level, such as the pronk and the trot, without the need of central feedback. The local feedback loop can make the system less dependent on the initial conditions from hop to hop, and more robust against disturbances, for example irregular terrain. Additionally, liftoff speed is easily measurable in a real robot, thanks to the joint position sensors, which are available in most robots.

Z.

3.This approach can simplify the complex natural behavior of the running machine, so that simpler high level controllers can make use of it.

This method was tested employing the DynaMechs simulation library (McMillan et al., 1996), and an accurate physical model of the KOLT robot. In simulation the position of the knee is controlled by a PD controller, so the knee joint behaves as if driven by a damped torsional spring. When the knee is maximally compressed during stance the desired position of the knee joint is changed instantly to a more extended position to produce additional thrust. The liftoff speed can be controlled in simulation with arbitrary precision by interrupting the thrust phase when the desired foot-hip speed has been reached. . To interrupt the thrust the leg is flexed for the next swing phase, even though the foot is still in contact with the ground.That is, when the desired liftoff speed has been reached, the leg is flexed rapidly, and since the body is already moving upwards this causes the foot liftoff. 


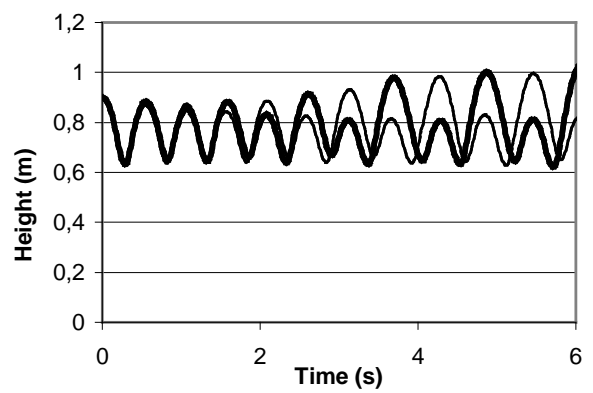

a)

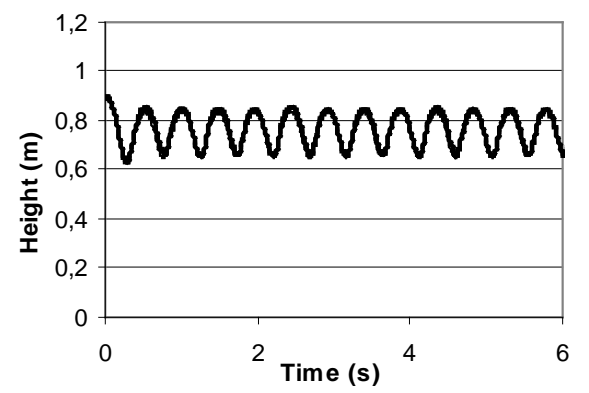

b)

Figure 3. Height of the fore and rear hips (thick and thin lines respectively) in a simulated pronk gait, a) without liftoff speed controller b) employing the liftoff speed controller.

Two simulations were run to prove the efficacy of this controller: in the first simulation the above described liftoff speed controller was not employed, i.e., thrust was not interrupted by the control system and the swing leg flexionphase was only started after foot liftoff. In the second experiment the liftoff speed controller was employed. Thrust was adjusted in both experiments to produce similar apex heights, and in both cases the robot was dropped with the body level and no angular speed. Figure 3Figure 3-a shows the behavior when this controller is not employed. As can be seen in thiln the first case (i.e., without liftoff speed control), ts graph, when the fixed thrust is employed the body pitch angle begins to oscillate-soon after the first hop, and, $\mathrm{t}$-being the period of the body pitch oscillation is twice as large as the hopping period (see Figure 3Figure 3Figure 3-a). The amplitude of the oscillation increases rapidly until it stabilizes after eight hops. Thus, the pronk and the bound gaits are not passively stable in the simulated robot, at least for the commanded hop-apex height. Figure 3Figure 3-b-In the second simulation, shows the behavior of thethe robot employing the liftoff speed controller while pronking. This graph shows how a pronk gait ean beis stabilized with the use of the simple closed loop liftoff speed controller, without the need of central feedback (see Figure 3Figure 3Figure 3-b). Other simple paired-foot gaits, in which the body remains predominantly leveled, such as the trot or the pace, can benefit from the same approach, at least to stabilize the body rotation about the pitching axis. This technique was implemented and tested in the KOLT robot as described in section 4.14.14.1. 


\section{Leg Thrust Control Methods}

Two methods have been employed to regulate leg thrust in the real KOLT robot. The first one implements the concept of liftoff speed control introduced in section $\underline{33} 3$ _, and the details of the implementation in the robot are described in section -4.1. Fand the second method makes use of a model of the electro-pneumatic thrusting system to control the amount of energy injected in each hop as a function of valve timing, and is presented in section 4.2 .

\subsection{Closed Loop Leg Thrust Control}

This controller regulates the liftoff speed with the use of direct sensory feedback of this magnitude, and thus its classification as closed loop. When the knee has reached its maximum compression point during stance, the valve is opened. The knee speed is then monitored during leg extension to estimate the liftoff speed. When the desired linear liftoff speed has been reached, the knee motor is actuated to wind up the cable and to cause the flexion of the leg, interrupting in this way the thrust phase.

To estimate the liftoff speed along the foot-hip direction $V_{\text {liftoff:, }}$ the knee position $\theta_{\text {knee }}$ is differentiated and filtered:

$$
V_{\text {lifooff }}(t)=\left(\alpha \frac{\theta_{\text {knee }}(t)-\theta_{\text {knee }}(t-1)}{T_{s}} L_{\text {link }} \sin \left(\frac{\theta_{\text {knee }}(t)}{2}\right)+(1-\alpha) V_{\text {lifioff }}(t-1)\right)
$$

$\mathrm{L}_{\text {link }}$ is the length of the shank and thigh links of the leg and $T_{s}$ is the sampling frequency. The parameter $\alpha$ of the first order filter was adjusted experimentally to obtain an accurate estimation. In order to do this, the liftoff speed estimated in real time in a hopping experiment was compared with a second estimate computed a posteriori from the measured time of flight (assuming a ballistic trajectory of a point mass located in the hip).

The principal difficulty for controlling the liftoff speed in the KOLT prototype is the fact that during the stance the knee motor cable is not under tension and is considerably slack. As a result, there is a time lag before the motor can effectively flex the leg to interrupt the thrust. This time lag depends on the angle of the knee (and thus the slackness of the cable) at the instant in which the desired liftoff speed is reached, making this effect difficult to compensate. Consequently, the actual liftoff speed beis always higher than the desired liftoff speed, as can be seen in the experimental results (section $\underline{5.105 .1)}$ ). 


\subsection{Open Loop Leg Thrust Control}

The second method proposed regulates the leg thrust by varying the instant at which the check valve is opened. Intuitively, the injected energy will be maximum if the valve is opened when the leg is maximally compressed, and no additional energy would be injected if the valve is opened at take-off. Thus, any intermediate amount of energy can be added opening the valve a certain time after the maximum leg compression and before take-off. Let $P_{\text {init }}$ be the initial pressure in the spring and reservoir when the spring is maximally extended, i.e., when its length is $L_{\max }$ and the leg is extended as well. During swing (leg phase 1), the knee motor flexes the leg,-so that the spring is-compressing the springed to a position-length $L_{\text {comp }}$, - while and the pressure in the spring and reservoir ascends to $P_{\text {comp }}$ given by:

$$
P_{\text {comp }}=P_{\text {init }}\left(\frac{V_{\text {res }}+V_{\text {par }}+A_{\text {cyl }} L_{\text {max }}}{V_{\text {res }}+V_{\text {par }}+A_{\text {cyl }} L_{\text {comp }}}\right)^{\gamma}
$$

where $A_{c y l}$ is the area of the piston $V_{\text {res }}$ is the volume of the reservoir, $V_{p a r}$ is the parasitic volume connecting the spring and the valve and $\gamma=1.4$ is the adiabatic exponent. When the spring is extended again to length $L_{\text {land }}$ in preparation for landing (leg phase 2), the pressure in the spring descends to $P_{\text {land, }}$, while the pressure in the reservoir remains constant_. Note that the volume in the reservoir is not considered in the calculation of $P_{\text {land, }}$ since-the check valve prevents the air in the reservoir returning to the spring):

$$
P_{\text {land }}=P_{\text {comp }}\left(\frac{V_{p a r}+A_{c y l} L_{\text {comp }}}{V_{p a r}+A_{c y l} L_{\text {land }}}\right)^{\gamma}
$$

After landing_(leg phase 3), the leg is compressed under the weight of the robotmentil it reaches the maximum compression point, storing energy in the spring in this process. Then it begins extending again, returning the elastic energy in the spring, until the valve is opened at spring length $L_{\text {valve }}$. When the valve is opened (leg phase 4), ideally the pressures in the reservoir and the spring equalize instantly_the pneumatic system was carefully designed to minimize transitory dynamics). The leg then continues extending until the initial state $\left(L_{\max }, P_{\text {init }}\right)$ is reached, returning the energy stored in the spring plus the additional energy stored during swing in the reservoir. The net additional energy injected in this process can be computed as the sum of the work done before valve opening 
Table 2. Characteristics of the pneumatic thrusting system.

\begin{tabular}{|l|l|l|}
\hline Pre-charge pressure & $P_{\text {init }}$ & $689 \mathrm{~K} \mathrm{~Pa}$ \\
\hline Reservoir volume & $V_{\text {res }}$ & $508 \cdot 10^{-6} \mathrm{~m}^{3}$ \\
\hline Parasitic volume & $V_{\text {par }}$ & $25 \cdot 10^{-6} \mathrm{~m}^{3}$ \\
\hline Spring piston area & $A_{\text {cyl }}$ & $1.828 \cdot 10^{-3} \mathrm{~m}^{2}$ \\
\hline Spring length at swing compression & $L_{\text {comp }}$ & $45 \cdot 10^{-3} \mathrm{~m}$ \\
\hline Spring length at landing & $L_{\text {land }}$ & {$[60,75] \cdot 10^{-3} \mathrm{~m}$} \\
\hline Maximum spring length & $L_{\text {max }}$ & $76 \cdot 10^{-3} \mathrm{~m}$ \\
\hline
\end{tabular}

(spring compression and beginning of spring extension), and after the valve opening (completion of spring extension): The net additional energy injected in this process is given by the work donemade by the spring during leg stance, which can be computed as the sum of the work before and after the valve opening:

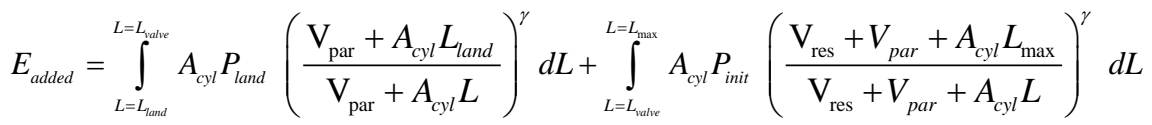

Please note that before valve opening, part of the energy stored in the spring during compression is returned during beginning of spring extension. First term of expression above represents the net energy stored in this process. Substituting (4) and (5) in (6) and integrating, the added energy is given by:

$$
\begin{aligned}
E_{\text {added }} & =\frac{P_{\text {land }}}{1-\gamma}\left(V_{p a r}+A_{c y l} L_{\text {land }}\right)^{\gamma}\left(\left(V_{p a r}+A_{c y l} L_{\text {valve }}\right)^{1-\gamma}-\left(V_{p a r}+A_{c y l} L_{\text {land }}\right)^{1-\gamma}\right) \\
& -\frac{P_{\text {init }}}{1-\gamma}\left(V_{\text {res }}+V_{p a r}+A_{c y l} L_{\max }\right)^{\gamma}\left(\left(V_{\text {res }}+V_{p a r}+A_{c y l} L_{\text {valve }}\right)^{1-\gamma}-\left(V_{\text {res }}+V_{p a r}+A_{c y l} L_{\max }\right)^{1-\gamma}\right)
\end{aligned}
$$

Thus, we can control the added energy modifying the magnitudes $L_{\max }, L_{\text {land }}, L_{\text {comp }}$ or $L_{\text {valve }}$. The first option, the variation of $L_{\text {max }}$, butld beis analogous to interrupting leg thrust before the leg is completely extended, so it is equivalent to the method presented in section 4.14.14.1. With tThe second and third options, have the advantage of storing only the energy that will be needed in the next stance is stored during the flight-during flight, either in the spring or in the reservoir, and this makes these methods more efficient. However, these options proved to be inaccurate methods-for regulating thrust, at least in our experimental system. Additionally, this method implies that the exact amount of 


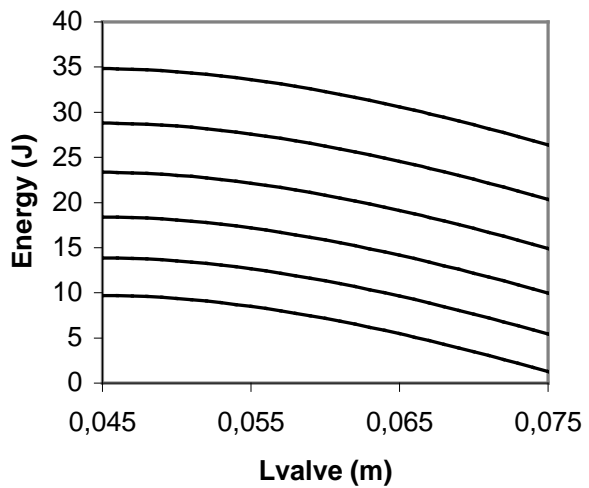

Figure 4. Energy added in a hop by the electro-pneumatic thrusting system as a function of the length of the spring when the valve is opened $\left(L_{\text {valve }}\right)$, for six different spring lengths at landing $\left(L_{\text {land }}\right)$, computed with the analytical model.

thrust needed in the next stance must be known at the beginning of the previous swing phase. For these reasons we adopted the third-fourth solution, the control of $L_{v a l v e}$ to finely regulate the energy injected, while $L_{l a n d}$ and $L_{\text {comp }}$ were simply pre-adjusted to store appropriate amounts of energy. This method has the additional advantage that the amount of energy injected in the each stance hop-can be decided as late as the maximum leg compression point of the stance, making possible the control of injected energy as a function of the leg compression to maintain the total energy (Raibert, 1986). -Figure 4 represents the energy injected in each hop as a function of $L_{\text {valve, }}$, for several values of $L_{\text {land }}{ }_{2}$ computed from the analytic model above and the characteristics of the KOLT robot depicted in Table 2Fable 2Table 2Table 2 . This graph shows that the injected energy that can be regulated with valve timing ranges from 0 to $10 \mathrm{~J}$, while the variation produced by the landing length ranges from 0 to $25 \mathrm{~J}$ and the total range that can be obtained is thus 35 $\mathrm{J}$.

In order to inject a particular amount of energy, the spring length can be monitored with the knee encoder, and the valve opened when the knee reaches the angle for which the injected energy predicted by the model is equal to the desired.

In order to inject a particular amount of energy, the spring length can be monitored with the knee encoder, and the valve opened when the injected energy predicted by the model equals the desired injected energy. A simpler method for energy regulation would be to open the valve a given time after the maximum compression; in this case the amount of 
energy injected would be unknown a priori, but it could be finely regulated, as can be seen in the next section.

\section{Experimental and Simulation Results}

\subsection{Two--Legged Bound Experiments}

In order to evaluate the performance of the two proposed thrust controllers, the KOLT robot was suspended by the rear part of the body, so that it could hop on its fore legs using them synchronously, as if they were one. These two legs support approximately half of the weight of the robot, so the conditions- were assessed experimentally to emulate-be very similar to those found in a four--legged pronk, in terms of leg compresion, hopping height and hopping period. To test the closed loop controller the target liftoff was set to $0.5 \mathrm{~m} / \mathrm{s}$ and increased $0.02 \mathrm{~m} / \mathrm{s}$ after each hop, during 40 consecutive hops. Figure 5Figure 5Figure 5Figure 7Figure 6 shows $_{2}$, for the 40 hops, the actual estimated liftoff speed estimated from the time of flight-as a function of the target liftoff speed. The liftoff speed is estimated a posteriori from the time of flight. This experiment shows the approximately linear relationship between the desired and actual liftoff speeds. Errors are larger for the lower
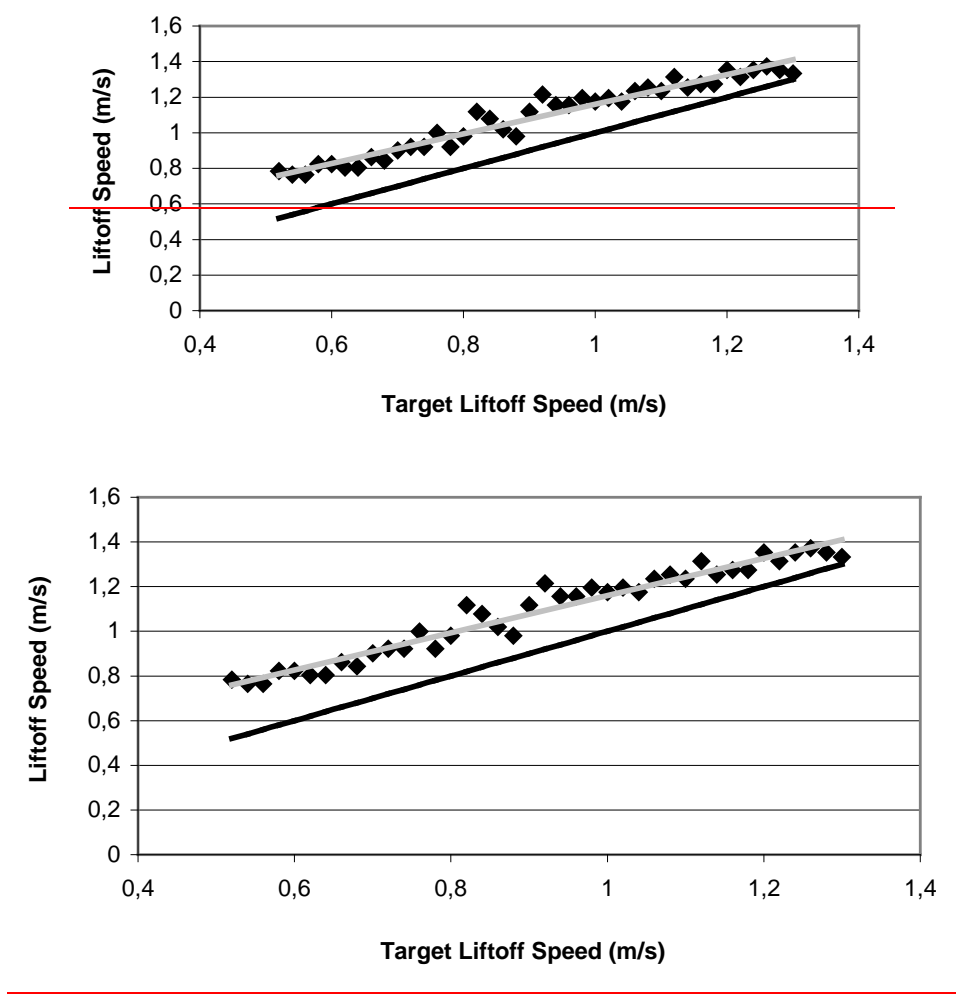

Figure 5. Actual liftoff speed estimated from the time of flight (rhombus) as a function of the target liftoff speed. A linear regression of the results (grey) and ideal behavior (black line) are also represented. 


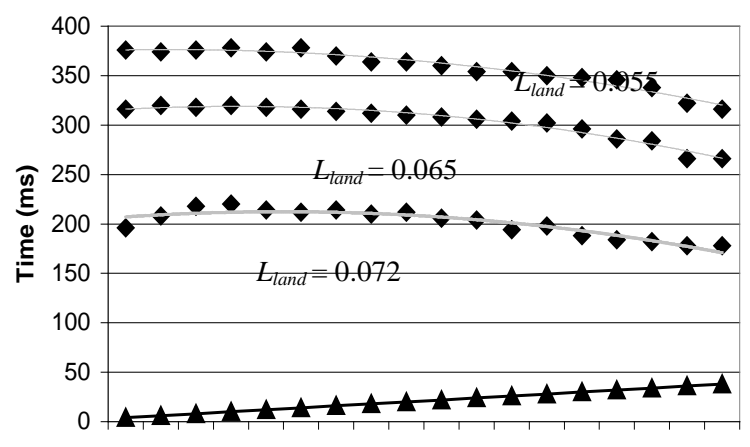

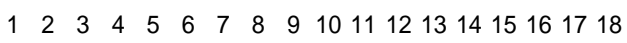

Hop \#

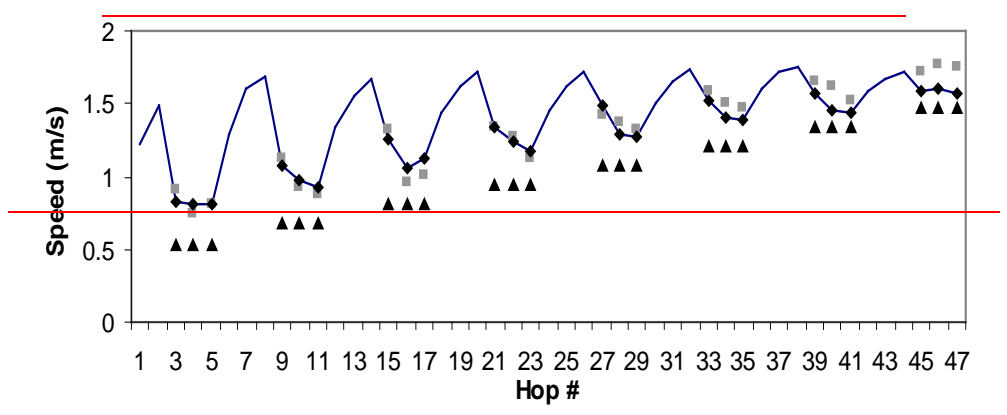

Figure 6. Times of flight (rhombus) obtained when the valve is opened a variable time after maximum leg compression (triangles), in 23 consecutive hops, for three different values of $L_{\text {land. }}$.

target speeds because the thrust must be interrupted when the leg is more flexed, and hence the cable is slacker. Absolute errors vary from 0.1 to $0.25 \mathrm{~m} / \mathrm{s}$ depending on the target liftoff speed, and relative errors vary from $7 \%$ to $19 \%$ when computed relative to the entire speed range $=(0$ to $1.4 \mathrm{~m} / \mathrm{s})$. This experiment shows that the liftoff speed can be continuously regulated, although positive errors will always be present.

Figure 665. Target liftoff speed (triangles), and liftoff speed estimated from knee encoder (fhombus and solid line) and from time of flight data (gray squares), in 48 consecutive hops

$$
\text { өf the KOLT robet. }
$$

In a second experiment, sets of three hops with maximm thrust were intercalated with sets of three hops with a controlled liftoff speed, resulting in abrupt changes in the working conditions. The aim of this test was to study the behavior in the transient state and to evaluate the robustness of the controller under varying target liftoff speeds and initiat eonditions. Figure 6Figure 6Figure 5 shows the target liftoff speed and two estimates of the 
liftoff speed obtained, caleulated from knee eneoder and time of flight data. This experiment shows that despite the time delay imposed by the mechanical characteristics of the leg, and even with changing initial conditions the liftoff speed can be controlled at least partially. The time between the activation of the motor and the foot take-off is below 16 ms. For a typical hip acceleration of $25 \mathrm{~m} / \mathrm{s}^{2}$ that causes maximum error of $0.4 \mathrm{~m} / \mathrm{s}$, in agreement with the results. Figure 6Figure 6Figure 5 shows that in the first hop after the maximum thrust hops the error is higher than in subsequent hops, because the legs are eompressed further after landing, producing a larger acceleration, and thus a larger error. Also the cable is slacker as a result of the further compression. The error in the estimation of liftoff speed was about $10 \%$, for liftoff speeds between 0.7 and $1.7 \mathrm{~m} / \mathrm{s}$. The delay introduced by the filter was $24 \mathrm{~ms}$ for the working sampling period $\left(T_{s}-2 \mathrm{~ms}\right)$. As a comparison, leg extension during stance lasts about $50 \mathrm{~ms}$ and $100 \mathrm{~ms}$ in pronk and trot gaits respectively. 


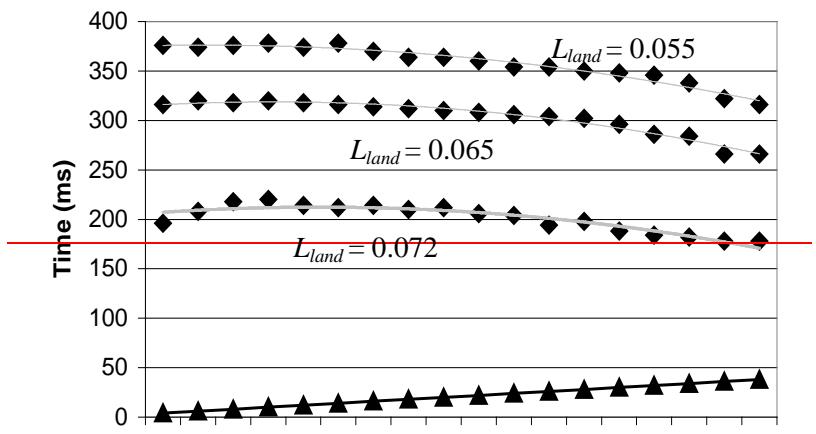

$\begin{array}{llllllllllllllllll}1 & 2 & 3 & 4 & 5 & 6 & 7 & 8 & 9 & 10 & 11 & 12 & 13 & 14 & 15 & 16 & 17 & 18\end{array}$

Hop \#

Figure 787. Times of flight (rhombus) obtained when the valve is opened a variable time after maximum leg compression (triangles), in 23 consecutive hops, for three different values of $L_{\text {tent. }}$

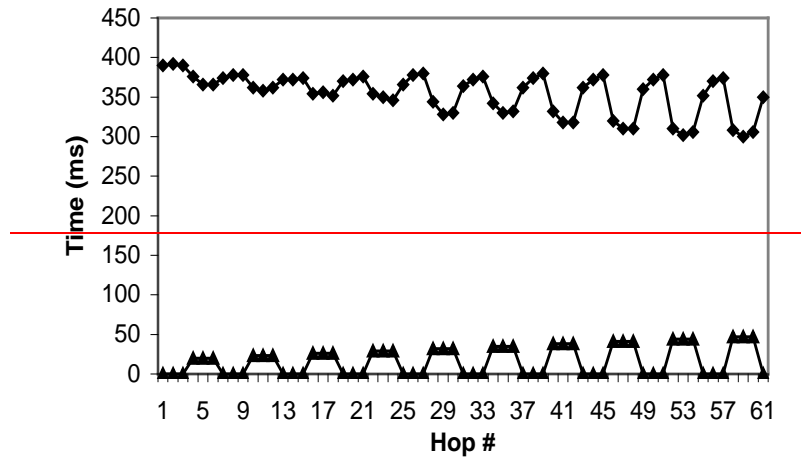

Figure 89 Times of flight (rhombus) obtained when the valve is opened a variable time after maximum leg compression (triangles), in 61 consecutive hops.

Similar experiments were run to test the open loop controller.- Figure 6 shows the times of flight obtained in 23 consecutive hops when the time from the maximum leg compression to the valve opening is increased $2 \mathrm{~ms}$ in each hop. The experiment was repeated for three different values of $L_{\text {land. }}$. The times of flight decrease as the valve time increases and $L_{\text {land }}$ increases, following a pattern that resembles the energy injected per hop represented in-Figure 4Figure 4, as explained in section 5.55.55.5. The range of times of flight that can be obtained with this method (from $77 \%$ to $100 \%$ of the maximum flight time) is much shorter than the range obtained with the closed loop controller (from 57\% to $100 \%$ of the maximum flight time). This is because the open loop controller can only regulate the additional energy injected from the reservoir to the system, while the closed 

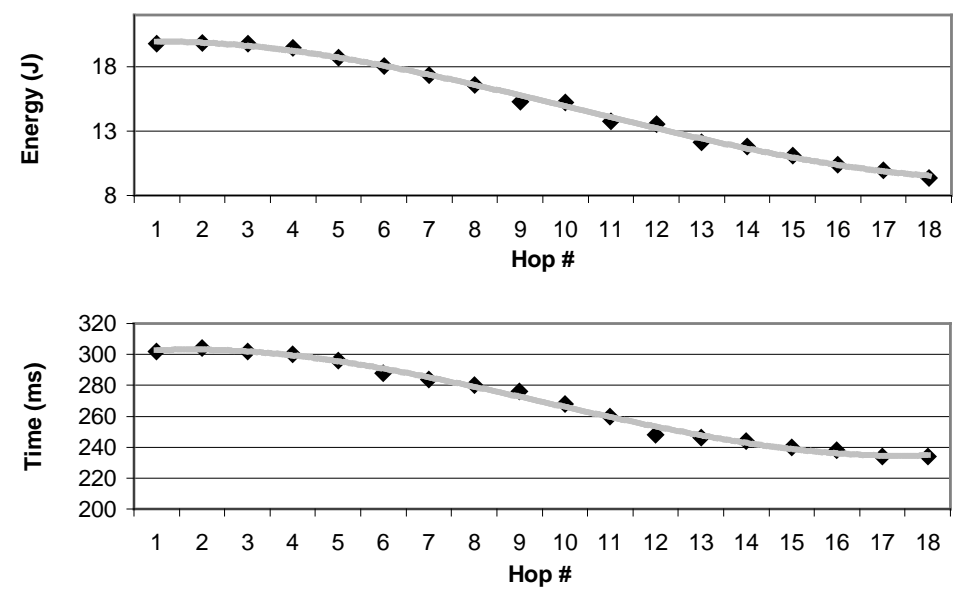

Figure 7. a) Desired injected energy b) Times of flight obtained.

loop controller can also regulate the energy stored in the spring that is injected to the system or even to dissipate energy. Figure 8 Figure 8 depicts the variable times of flight obtained when the valve timing was varied in sets of three hops showing the variation of the hop height when the energy injected changes abruptly.

Another experiment was run to test the possibility of injecting a specific amount of energy in each hop by opening the valve when the spring reaches a prearranged length. Figure 7Figure 11Figure 9Figure 9 shows the desired injected energy, calculated by the model, and the times of flight obtained. Although in this experiment it is not possible to verify if the energy predicted by the analytic model is actually injected into the system (since most of the injected energy is employed to compensate losses), this test is useful to describe the relation between the injected energy and the system losses, as explained in section $\underline{5.55 .55 .5 .}$

\subsection{Pronk simulation}

Four simulations have been run to illustrate the advantages of the closed loop controller to stabilize the body motion even in the presence of disturbances such as irregular terrain or changes in the forward speed target. In these experiments no leg coordination mechanisms were implemented to force bound or pronk gaits. 


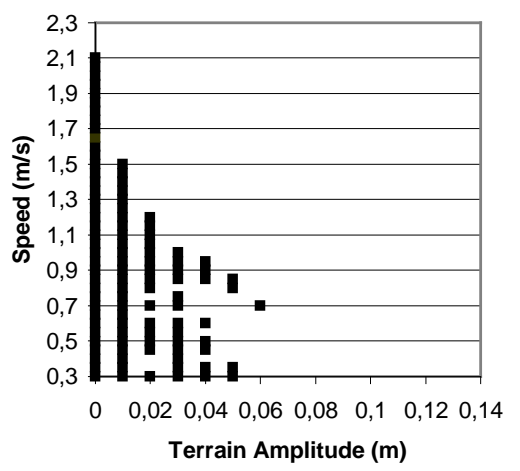

a)

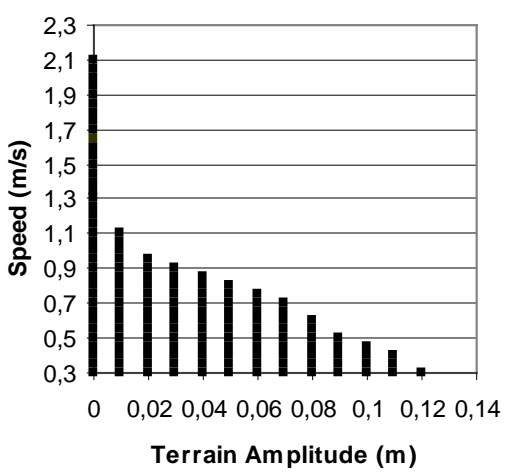

b)

Figure 9. Pronk simulation: Succes (black squares) or failure to traverse a sinusoidal terrain with different amplitudes at several speeds a) with the open loop thrust controller b) with the closed loop thrust controller.

In the first simulation the robot runs on flat terrain at several forward speeds between 0.3 $\mathrm{m} / \mathrm{s}$ and $2 \mathrm{~m} / \mathrm{s}$, and the apex height is $0.11 \mathrm{~m}$. Figure 8Figure 10Figure 10Figure 10 shows the peak oscillations in body pitch with the open loop and the closed loop controllers. It is apparent that higher speeds have a stabilizing effect on the body pitch (an effect-observed in the real machine as well), so the oscillations remain within reasonable levels with both controllers for speeds above $1 \mathrm{~m} / \mathrm{s}$.

However, at low speeds only the closed loop controller is successful in stabilizing the body and it becomes indispensable in order to obtain practical locomotion.

In the second simulation, the robot runs on irregular terrain, with the same apex height. The terrain section is sinusoidal, and the wave length is twice the distance between hips, so that

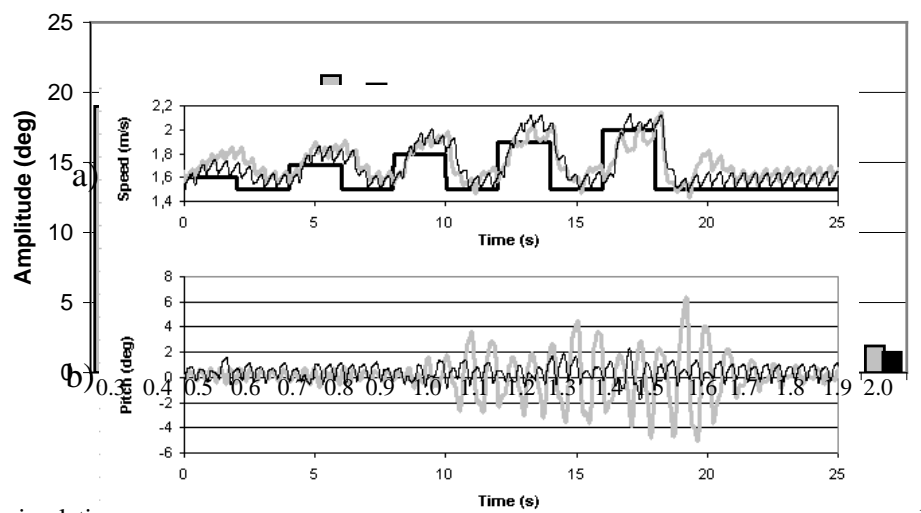

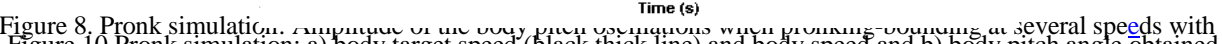

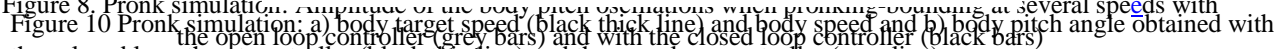
thee closed loop thrust controller (black thin line) and the open loop controller (grey line). 
the fore feet can eventually land on a crest while the rear feet land on a valley. Several sine amplitudes have been employed ranging from flat terrain to $\pm 0.12 \mathrm{~m}$. Figure 9Figure 13Figure 12Figure 12 shows, for both controllers, the success or failure when traversing irregular terrain with different irregularity and with different speeds. With the use of the closed loop controller the robot is able to traverse terrain with twice the irregularity amplitude than with the open loop controller, although lower speeds must be employed. According to the graph, the open loop controller seems to be more successful in smooth terrain; however, the amplitude of the body oscillations (even larger than those described in the first simulation) again makes locomotion impractical without the closed loop controller. The third simulation illustrates the ability of the closed loop controller to stabilize the body against changes in speed. Figure 10Figure 12Figure 11Figure 11 shows the target speed profile, with several abrupt changes. Although both the closed and the open loop controllers offer similar results for speed tracking, the closed loop controller is clearly superior in stabilization of the body, reducing pitch oscillations by a factor of three.

In the fourth simulation the robot pronks in place on flat terrain. The pronk was passively stable for apex heights lower than $0.06 \mathrm{~m}$, and thus the open loop controller was useful only in this region. However, the closed loop controller could stabilize the gait for higher apex heights. Figure 11Figure 14Figure 13Figure 13 shows the average mechanical power employed to maintain the stable pronk beyond the passive stable region (up to 2.5 times the maximum passively stable apex height). Mechanical power is computed multiplying torques and angular speeds of each joint. To offer a comparison, this graph also shows the average power used by the open loop controller when a level body posture is forced in the simulation to achieve stability (fby restricting the degrees of freedom of the body. mAotion is restricted to a plane and body rotations are not allowed). This simulation shows how stability can be achieved at the cost of higher energy expenditure.

It has to be noted that since two dimensional models are employed (there is no distinction between right and left legs), simulation results for the- pronk are extensible to the trot gait. 
The pronk gait was tested in two experiments with the KOLT robot employing the two leg thrust controllers: the closed loop liftoff speed controller and the open loop controller. Preliminary tests were made in order to find target values for both controllers that resulted in similar times of flight so that the results of the experiments could be compared. In the case of the liftoff speed controller the target liftoff speed was set to $1.0 \mathrm{~m} / \mathrm{s}$. In the case of

a)

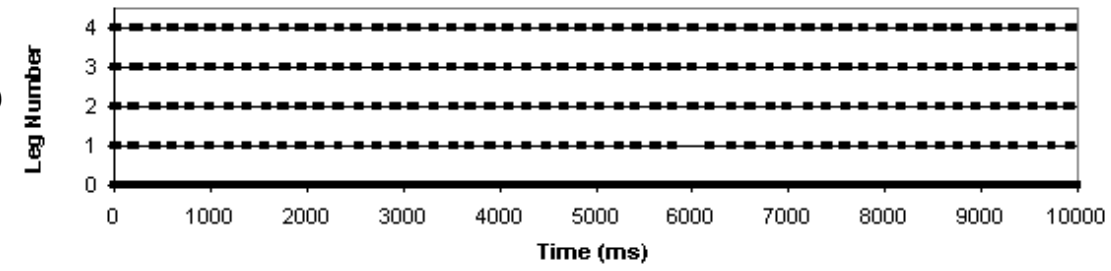

b)

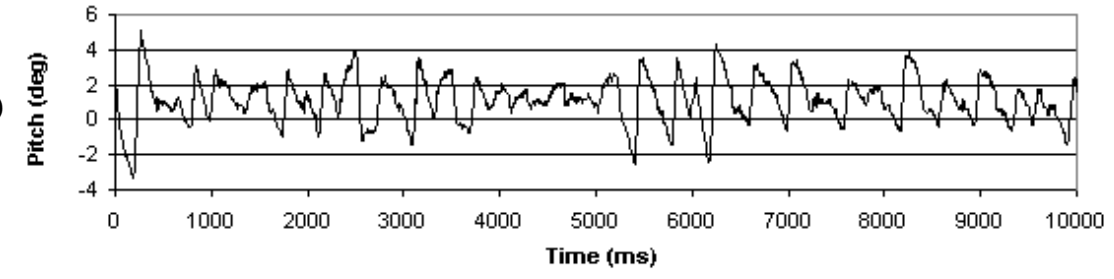

c)

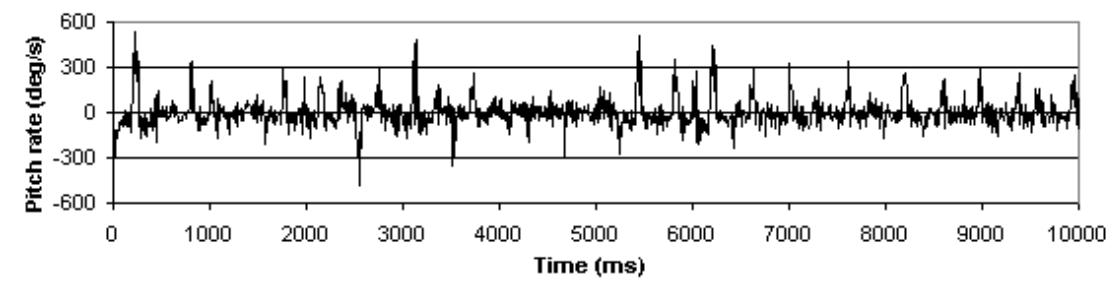

d)

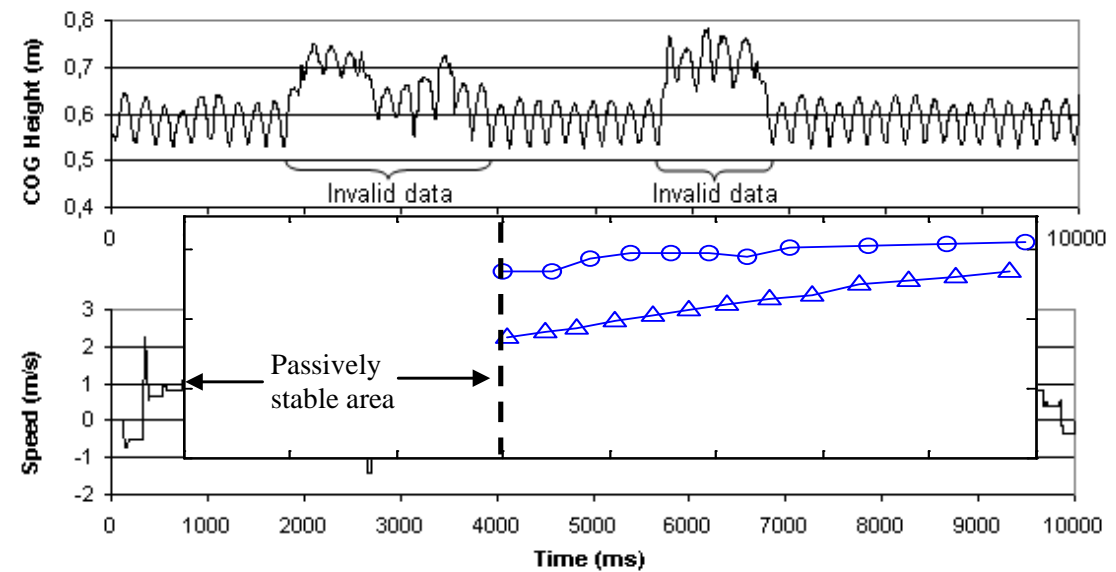

Figure 11. Mechanical pPower employed to maintain a pronk beyond the passively stable region as a function of the

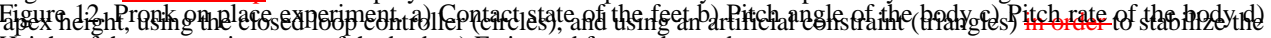
Height oftbaigeometric center of the body e) Estimated forward speed. 
the open loop controller the valve was opened at the bottom of flight and $L_{\text {comp }}$ and $L_{\text {land }}$ were pre-adjusted to $0.045 \mathrm{~m}$ and $0.073 \mathrm{~m}$ respectively. The desired forward speed for the robot was set to zero. The robot runs freely on the treadmill with its motion confined to a plane, as explained in section $2 z 2$.

Figure 12Figure 15Figure 14Figure 14 shows the foot contacts, the pitch angle and rate, the COG height and the estimated forward speed obtained using the closed loop liftoff speed controller. The KOLT robot performed a stable, almost periodic pronk, with minor pitch excursions. The standard deviation of the pitch around the average value is only 1.24 degrees, and maximum pitch excursions are about 7 degrees. The pitch angle signal shows a characteristic saw shape: when a pair of legs (the front or the rear legs) lands slightly before than the other, a fast body pitching motion appears. This motion is consequence of both the high stiffness of the legs and the fact that the length of the legs is not adjusted to synchronize their landings (Raibert, 1990), since this would vary the energy stored in the springs. The high stiffness of the legs is a result of the high pre-charge pressure in the pneumatic system required to store enough energy during flight. As a consequence, the duty factor was only 0.22 , corresponding to an average support time of $62 \mathrm{~ms}$ and a flight time of $218 \mathrm{~ms}$. This flight time implies a liftoff speed of $1.060 \mathrm{~m} / \mathrm{s}$, very close to the target Assuming ballistic conditions, this flight time implies a liftoff speed of $1.060 \mathrm{~m} / \mathrm{s}$, very close to the target. However, as stated above flight is not purely ballistic due to asynchronous landing of the legs and body dynamics.-

Figure 13Figure 16Figure 15Figure 15 shows the pitch angle in a pronk experiment employing the open loop controller. In this case the robot performed a mixture of pronk and bound gaits resulting in an irregular motion with large pitch excursions, showing

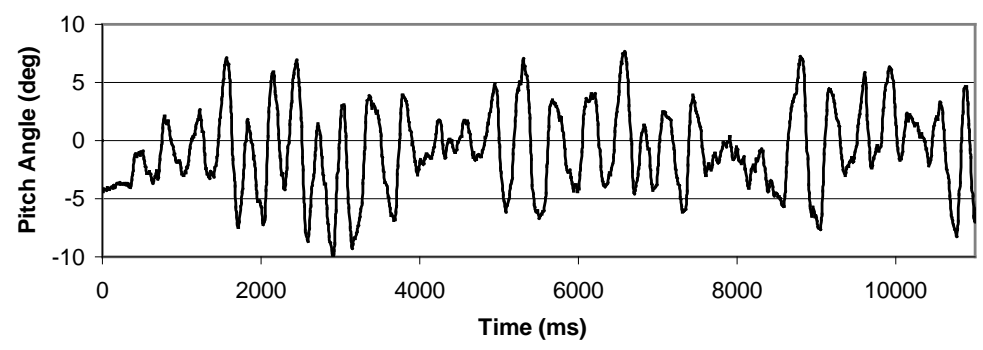

| Figure 13. Pronk on place experiment: Pitch angle measured when the open loop controller is employed to-regulates leg thrust. 
similar results to those obtained in simulation (see section $\underline{33} 3$ ). The standard deviation of the pitch around the average value is 3.55 degrees and the maximum pitch excursions are above 15 degrees. Comparing the results ebtained inof both experiments it can be concluded that the liftoff speed controller helps to actively stabilize the passively unstable pronk, reducing considerably the pitching motion.

\subsection{Trot Experiments}


In the trot experiments the KOLTrobet runs freely on a treadmill at $1.1 \mathrm{~m} / \mathrm{s}-\underline{\text { with }}$ its motion confined to a plane, as explained in(see section 222). The liftoff speed controller was employed and the target speed was set to $1.0 \mathrm{~m} / \mathrm{s}$. Figure 14Figure 17Figure 16Figure 16 shows the experimental data describing the gait.

Compared with the pronk gait, the trot is more stable as a result of the more compliant behavior obtained when landing on two legs instead of on four. The lower total stiffness of

a)

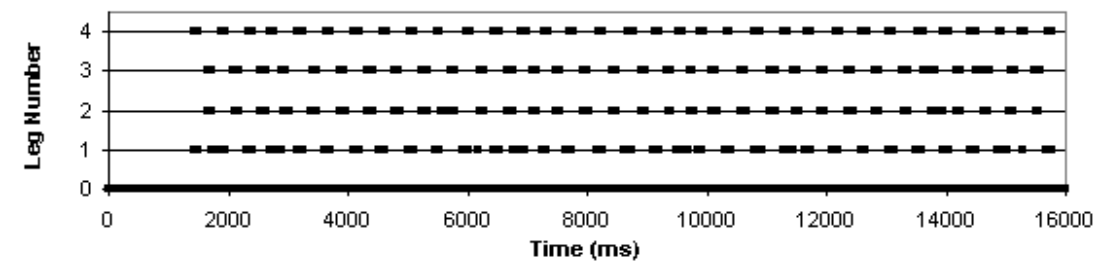

b)

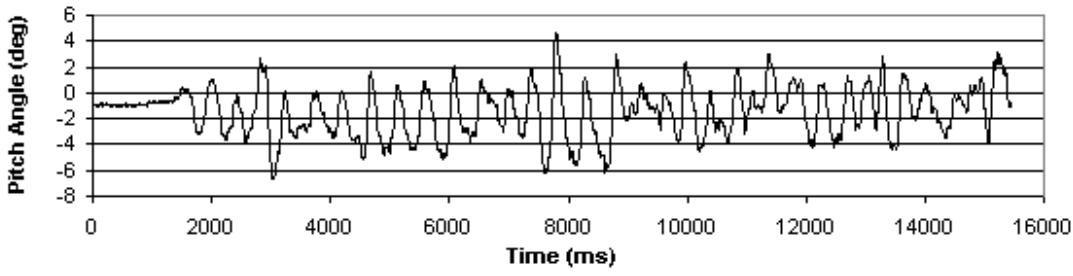

c)

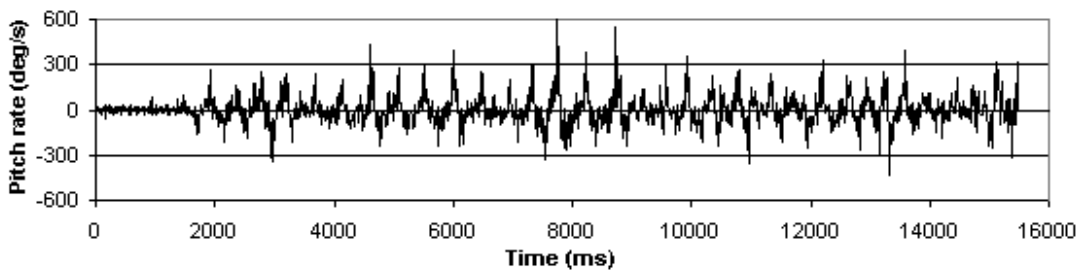

d)

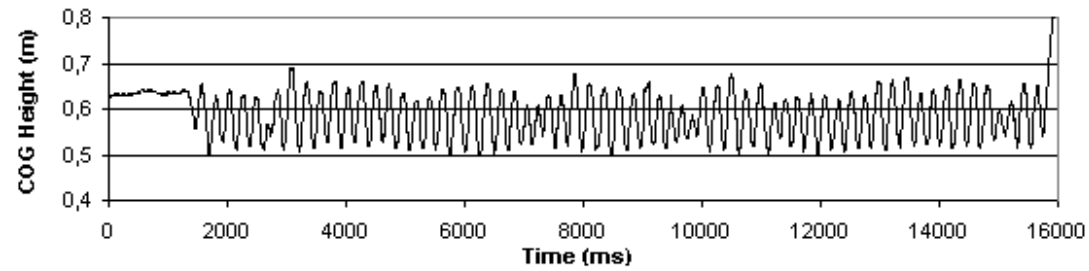

e)

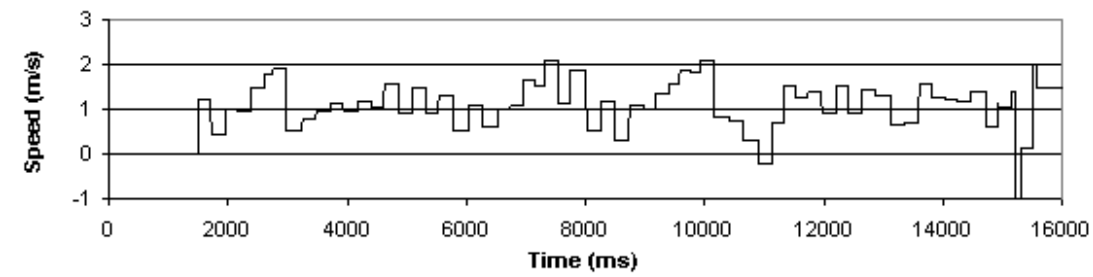

Figure 14. Trot experiment. a) Contact state of the feet b) Pitch angle of the body c) Pitch rate of the body d) Height of the geometric center of the body. e) Estimated forward speed. 
the landing legs smoothes the pitch motion of the body, accommodating attitude errors at landing without causing high rate pitch motions. As a result, the pitch signal is more similar to a sinusoidal function than to a saw function, and the machine is in contact with the ground approximately $43 \%$ of the time, corresponding to a support time of $157 \mathrm{~ms}$ and a total period of $740 \mathrm{~ms}$. The duty cycle observed is again-certainly low if compared with quadruped mammals for which the total duty factor (i.e., the sum of the durations of leg stances divided by the stride period) declines with increasing speed from about $75 \%$ to $50 \%$ in a fast gallop, and trotting duty factors are substantially longer (Gambaryan, 1974).

The variable estimated speed is due partly to errors in the estimation and partly to the robot actually moving forward and backwards on the moving treadmill. This last case is reflected also in the lower COG height observed when the robot moves faster (near $t=10 \mathrm{~s}$ for example). In these experiments the body pitch angle oscillates with a period twice as large as the hop period. This effect was predicted by the simulation (see section $\underline{33}$ ) and is a consequence of the high moment of inertia of the robot about its pitching (transversal) axis.

\subsection{Power, Energy and Efficiency Experiments}

The energetics of locomotion have been studied in two experiments with the KOLT robot in-order-to provide insight into general aspects of locomotion, such as the power effectively employed to sustain the motion or and the impact losses, and also particular design aspects_of the of the prototypeKOLT, such as the efficiency of the motors, mechanisms and power electronics.

The energetic efficiency of the thrusting system was analyzed firstduring-separately in the two--legged bound experiment with open loop controller experiment described inf(see section 5.105.12. Figure 18Figure 15Figure 17Figure 17 shows the energy injected per hop and per leg calculated with the model, as a function of the apex height. Since the variation in apex height during the experiment is slow, the energy added per hop ean-approximates, as an upper bound, the energy needed to maintain a given hop height. Thus, the energy injected is an approximation for the losses, which are caused mainly by the foot/ground impacts and secondarily by friction in the joints and in the spring. Figure 18Figure 15Figure 17Figure 17 shows elearly a linear relation between the losses and the apex height in the practical hop height range. The mechanical work executed done by the motors, $W_{m}$, 


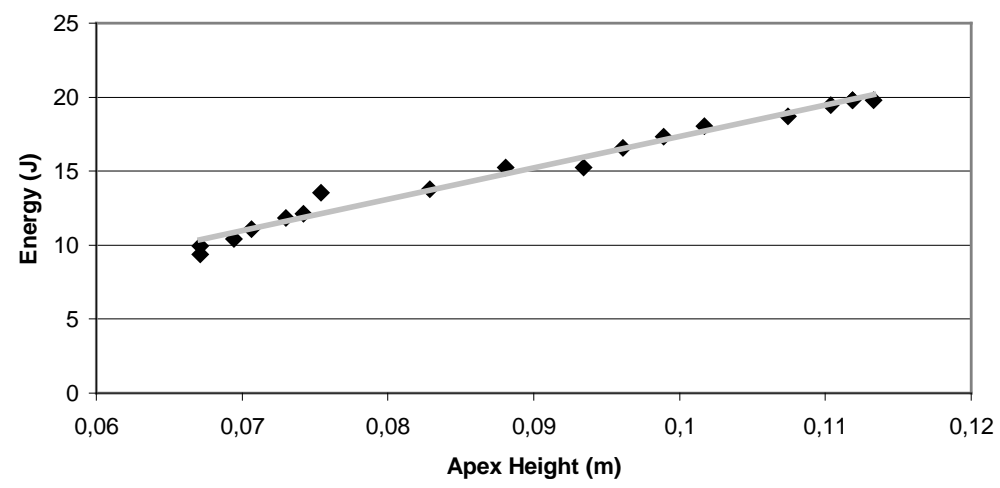

Figure 15. Energy injected per leg and hop, $\mathrm{W}_{\mathrm{W}_{2}}$ as a function of the apex height in a two--legged bound.

Formatted: Subscript was calculated integrating the mechanical power, $\mathrm{P}_{\mathrm{m}_{2}}$ over the leg cycle period $T$. $\underline{\text { The }}$ mechanical power is computed as the product of motor torque $\tau_{m}$, and shaft speed, $\omega$ :

$$
W_{m}=\int_{t=0}^{t=T} P_{m}(t) d t=\int_{t=0}^{t=T} \tau_{m}(t) \omega(t) d t
$$

The torque was $\mathrm{C}$-computing $\tau_{m}$ ed from the measurements of the current in the motor winding. According to this,, the mechanical work measured was estimated to be $\underline{W}_{m}=96 \mathrm{~J}$ per leg and per bounce. The electric energy consumed, measured in the input to tincluding losses in the power drives, and thus accounting for the whole energy expenditure_-was $\underline{\mathrm{W}}_{\mathrm{e}}=$ $155 \mathrm{~J}$ per leg and per-bounce. Then,-tThe efficiency of the power drive-actuator set, iscalculated as the ratio of electric power to mechanical power, is $\underline{W_{m}} / W_{\underline{e}}=$ consequently $61 \%$. Both the electric and the mechanical work $\underline{W}_{\text {e }}$ and $\mathrm{W}_{\mathrm{m}}$ per hop rremain almost constant despite the variation in the apex height. According to the model ${ }_{2}$ the energy injected-per hop, $\mathrm{W}_{\mathrm{i} 2}$ ranges from $9 \mathrm{~J}$ to $20 \mathrm{~J}$ (see Figure 15Figure 18Figure 17Figure 17) and eonsequently then the efficiency of the mechanical-actuator system (computed as $\underline{\mathrm{W}}_{\mathrm{i}} / \mathrm{W}_{\mathrm{m}} \mathrm{L}$ ranges from $9 \%$ to $20 \%$. This is due to friction in the joints and transmissions and principally toby the fact that part of the energy stored in the spring when the motor compresses it (i.e., during phase 1, see Section $\underline{22} 2$ ) is lost when the leg is extended in preparation for landing (i.e., during phase 2). The total efficiency of the thrusting system, i.e., the fraction of energy input that is effectively employed to maintain the bounce computed as injected energy divided by the electric work is up to $\underline{\text { is }} \mathrm{W}_{\underline{\mathrm{i}}} / \mathrm{W}_{\underline{\underline{e}}}=12 \%$ 
maximum., and it corresponds to the fraction of energy input that is effectively employed to maintain the bounce.

More general energetic aspects of the locomotion were also analyzed in the trot experiment described in section 5.45.45.4. In order to illustrate the magnitude of the energies involved in the trot gait and their evolution, helping to put in perspective the energy consumption and efficiency of the machine, Figure 16Figure 19Figure 18Figure 18 depicts the kinetic, potential and elastic energies during the trot experiment. This helps to put in perspective the energy consumption and efficiency of the machine.- To study the efficiency of the actuator systems Figure 17Figure 20Figure 19Figure 19 shows the mechanical power output, $\underline{P}_{\underline{m}_{2}}$ and the torque ${ }_{2} \underline{\tau}_{\underline{m}}$, of the knee motors, while Figure 18Figure 21Figure 20Figure 20 shows the electric power, $\underline{\mathrm{P}}_{2}$ consumed by the knee and the thigh motors-of a leg. Finally Table 3Table 3Table 3Table 3 shows the average and peak mechanical and electric power $\underline{P}_{m}$ and $\mathrm{P}_{\underline{e}}$ in the knee and thigh-motors during the trot, as well asand the efficiency of the actuators systems-(including composed by the-power electronics-and the motors) and of the whole quadruped.

The analysis of the above presented results yields several conclusions.-Two issues distinguish energetically the trot and the pronk (or the equivalent two--legged bounce) in the KOLT robot. First, Iin the trot, the knee motor must keep the swing leg flexed (and thus the spring compressed) in order-to produce ground clearance during the stance of the contralateral leg. As can be seen in Figure 20Figure 19 Tthe knee motor must exerts a considerable torque without producing any work during flight_see Figure 17Figure Z0Figure 19). Second, as a result of the limited range of motion of the knee theAlso, the thigh must be moved quickly during swing to a forward position (30 degrees

Table 3: Energetic consumption and efficiency in a trot gait.

\begin{tabular}{|l|l|l|l|}
\hline & Knee & Thigh & Total (4 legs) \\
\hline Average Electric Power (Average $\left.\mathrm{P}_{\mathrm{e}}\right)$ & $325 \mathrm{~W}$ & $196 \mathrm{~W}$ & $2084 \mathrm{~W}$ \\
\hline Peak Electric Power (Peak $\left.\mathrm{P}_{\mathrm{e}}\right)$ & $1300 \mathrm{~W}$ & $1200 \mathrm{~W}$ & $7600 \mathrm{~W}^{*}$ \\
\hline Average Mechanical Power (Average $\left.\mathrm{P}_{\mathrm{m}}\right)$ & $133 \mathrm{~W}$ & $122 \mathrm{~W}$ & $1020 \mathrm{~W}$ \\
\hline Peak Mechanical Power (Peak $\left.\mathrm{P}_{\mathrm{m}}\right)$ & $1100 \mathrm{~W}$ & $1100 \mathrm{~W}$ & $6600 \mathrm{~W}^{*}$ \\
\hline Electric Work per Step/Cycle $\left(W_{e}\right)$ & $243 \mathrm{~J}$ & $147 \mathrm{~J}$ & $1560 \mathrm{~J}$ \\
\hline Mechanical Work per Step/Cycle $\left(W_{m}\right)$ & $99 \mathrm{~J}$ & $92 \mathrm{~J}$ & $764 \mathrm{~J}$ \\
\hline Efficiency of actuator systems $\left(W_{m} / W_{e}\right)$ & $41 \%$ & $62 \%$ & $49 \%$ \\
\hline Specific resistance at $(1.1 \mathrm{~m} / \mathrm{s},(\varepsilon)$ & - & - & 1.18 \\
\hline
\end{tabular}

\footnotetext{
* Estimated values
} 
approximately)-to provide additional ground clearance. The thigh is moved to the required touchdown angle prior to landing, and both thigh movements must be done quickly to avoid unwanted ground contacts. These issues make-increase the power consumption significantly higher than-in the pronk (or the equivalent two legged bounctroet). However, due to the nature of thigh swing motion, the thigh energy consumption caused by these issues is not expected to increase with the vehicle speed. Also, the experimental results presented above suggest that knee motor consumption will not increase significantly either, so the energetic efficiency of locomotion-is expected to increase noticeably with theat higher speed. On the other hand,Also, both problems could be solved in future designs by the use of an additional joint in the shank, below the knee, which would also help to produce enough ground clearance with almost no additionalreduced energetic cost.

In both experiments the efficiency of the actuatorsactuators $\left(W_{m} / W_{e}\right)$ is around $50 \%$, a typical value in robotic systems working in start-stop regimes. The similar mechanical power consumption in the thigh and knee actuators in the trotis a finding comparable to the results obtained by Ahmadi and Buehler (1999) at almost the samesimilar speed $(1.2 \mathrm{~m} / \mathrm{s})$. The electric power consumption-_is a new result indicating that knee motors consume considerably more energy than the thigh motors in our experimental platformthe KOLT. The results show that differences between the-peak and average-electric power consumption ( 3 to 1 approximately)-could be diminished significantly by reducing the acceleration and speed of the joints to the values strictly required by the locomotion. . This kind of optimization would help towards the development of an energetically autonomous vehicle.

The specific resistance is a measure of the energetic efficiency usually employed to compare the performance of different kinds of vehicles, and particularly legged robots (Ahmadi and Buehler, 1999). The specific resistancelt is defined as the ratio of power output, $P_{0^{-}}$and the product of vehicle speed ${ }_{2} v_{f}$ and vehicle weight, $m g$ : 


$$
\varepsilon=\frac{P_{o}}{m g v_{f}}
$$

In the KOLT robot, the specific resistance is $\underline{\varepsilon}=1.18$ for a speed of $\underline{\underline{\varepsilon}} 1.1 \mathrm{~m} / \mathrm{s}$, $\underline{i}$ when $t \underline{f}$ the average mechanical power output (average $\mathrm{P}_{\underline{m}}$ ) calculated above is considered. Then,If eompared with other legged vehicles-it can be stated that the KOLT robot is the most efficient multi-legged machine according to the classification offered by Ahmadi and Buehler (1999). Some relevant multi-legged machines to compare are the ASV walking hexapod ( $\varepsilon \approx 1$ for $v_{f} \approx 1 \mathrm{~m} / \mathrm{s}$ ) (Pugh et al., 1990) and Raibert's running quadruped ( $\varepsilon \approx 10$ for $v_{f} \approx 1 \mathrm{~m} / \mathrm{s}$ ). Among the legged machines-Tthe efficiency of the KOLT robot is only outperformed by the ARL-II monopod ( $\varepsilon=0.7$ for $v_{f}=1.2 \mathrm{~m} / \mathrm{s}$ ), the most energy-efficient, actively-powered legged robot according to this classification. However, it has to be eonsidered that the KOLT robot has not been tested at its maximum speed yet (it was designed to run at $5 \mathrm{~m} / \mathrm{s}$ ) and power-energetic efficiency consumption-is-not expected to

a)

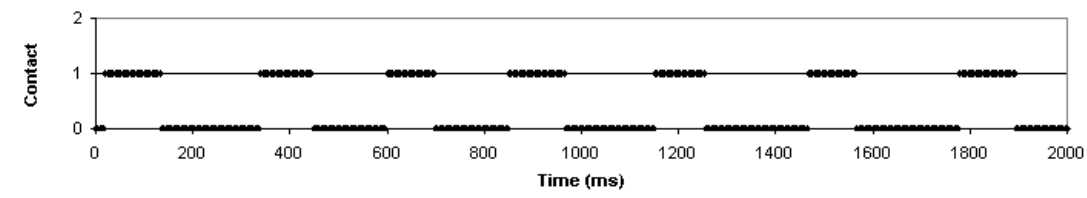

b)

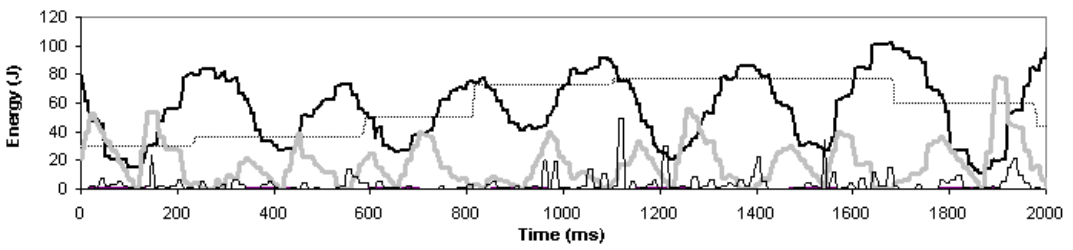

c)

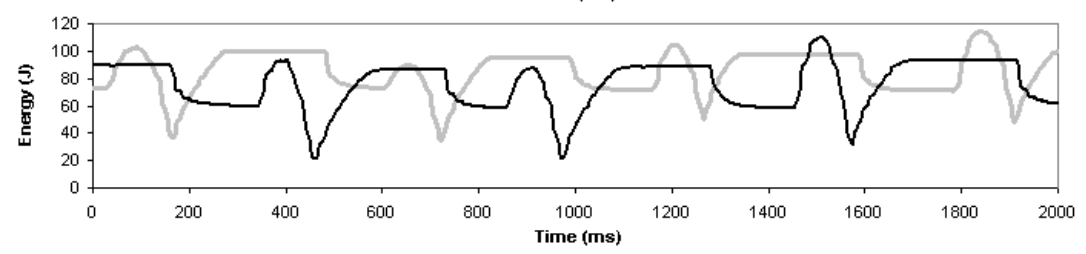

d)

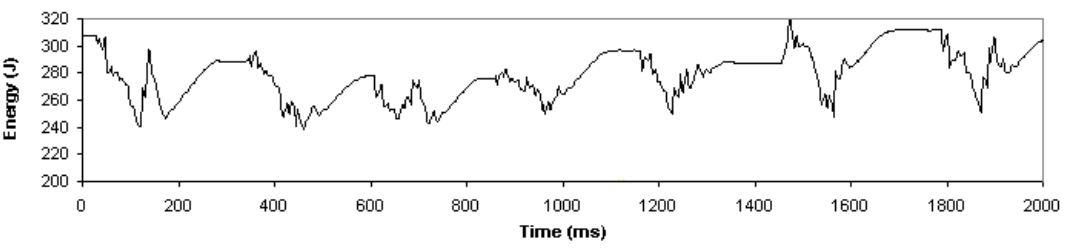

Figure 16. Trot experiment: a) Ground contact state of the robot b) Potential energy (thick black line), kinetic vertical energy (grey), horizontal kinetic energy (dotted) and kinetic rotational energy (thin black) c) Elastic energy of the two pairs of diagonal legs (black and grey) d) Sum of all the previous energies. 
a)

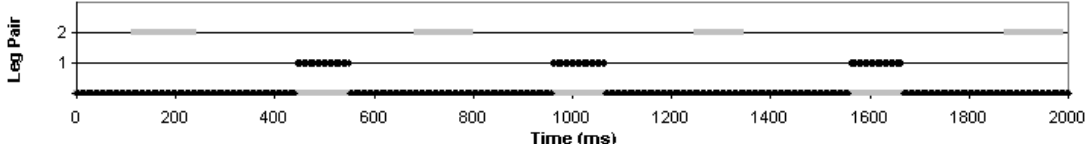

b)

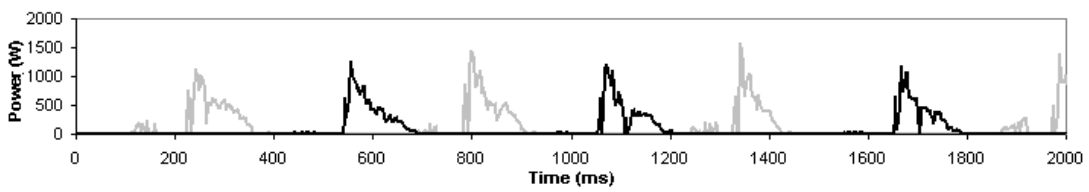

c)

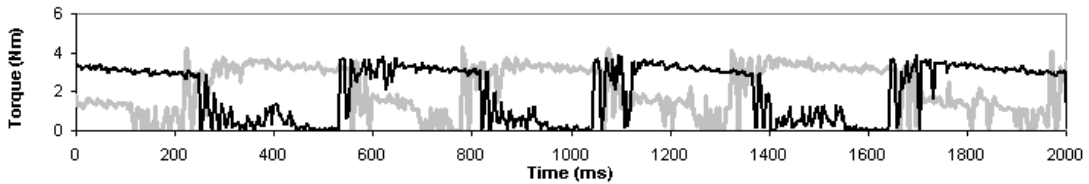

Figure 17. Trot experiment: For the two rear legs (grey and black lines): a) Contact state of the feet b) Mechanical work power $\mathrm{P}_{\underline{\mathrm{m}} \_}$of the knee motors c) Torque $\underline{\underline{\tau}}_{\underline{\mathrm{m}}}$ of the knee motors

increase significantly at higher speeds based on thee extrapolation of the observed behavior. Thus, the use of higher speeds and the power saving techniques suggested above eould increase significantly the efficiency in the near future. HoweverNevertheless, further refinements of the already efficient mechanical design will be the key towards an energetically autonomous running robot.

\section{Conclusion}

a)

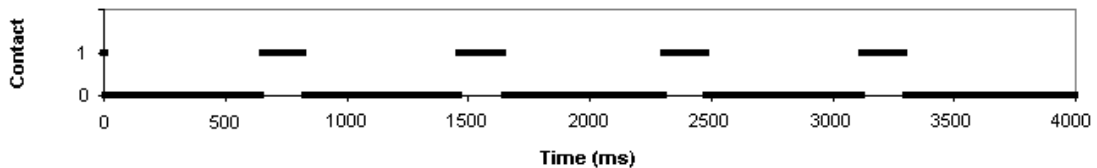

b)
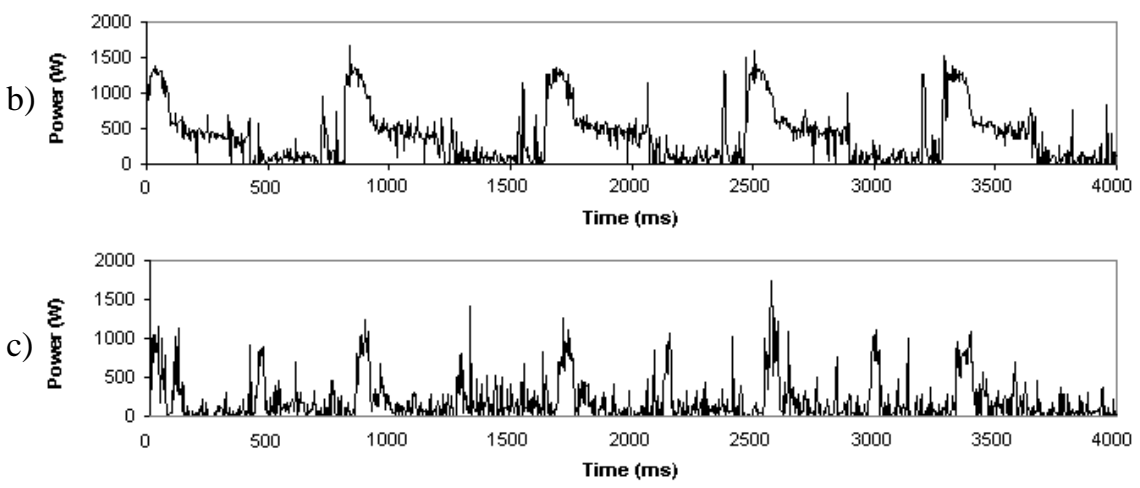

Figure 18: Trot experiment: a) Contact state of the foot b) Electric power consumption ${ }_{2} \underline{\mathrm{P}}_{\underline{e}}$ in the knee motor b) Electric power consumption, $\underline{\mathrm{P}}_{\underline{\underline{e}}}$ in the thigh motor. 
This paper deals with the design of energetically efficient actuators, suited to the demands of fast legged locomotion, and the control of these actuators to achieve stable running gaits. A simple closed loop controller has been proposed here to regulate the leg thrust and to stabilize the body pitching motion in planar pronk and trot gaits. Despite its simplicity and its limited accuracy in our prototype, this low--level controller is able to stabilize an inherently unstable gait (i.e. with an apex height above $0.06 \mathrm{~m}$ in the KOLT robot) without employing any central feedback, a property that can help to simplify the sensor system and obtain more robust gaits. Both simulations and experiments show the validity of this approach even on irregular terrain. Additionally, an energetic model of the novel leg thrusting mechanism of the KOLT robot has been developed and analyzed to determine and control the-amount of energy injected on each hop as a function of several control parameters. An open loop thrust controller based on this model has been also presented and tested experimentally. The pronk and trot experiments performed employing both controllers have permitted the analysis of the dynamic behavior of the quadruped, especially the stability of its pitching motion. The speed reached with the trot $(1.1 \mathrm{~m} / \mathrm{s})$ is close to the fastest electrically driven running robot $(1.2 \mathrm{~m} / \mathrm{s})$ and is expected to be increased soon. Finally, the electric power consumption, the mechanical work, and the state of the robot (height, speed, spring length, etc.) have been studied experimentally allowing the complete characterization of the energetics for the first time in an electrically actuated quadruped running robot at this scale $(80 \mathrm{Kg})$. Some relevant results of this energetic study are the efficiency of the actuators (49\%) and the total efficiency of the thrusting system (22\%). Additionally $y_{2}$ it has been found that ground impact losses are approximately linearly related with the apex height. The calculated specific resistance (1.18) makes the KOLT robot one of the most efficient multi-legged machines built to-date and indicates the validity of its efficiency oriented mechanical design date.

However, the effectiveness of these mechanical designs and control strategies has only been tested in planar pronk and trot gaits with limited forward speed (about $1.1 \mathrm{~m} / \mathrm{s}$ ) and on planar terrain. Future work includes the integration of these control techniques in more complex controllers in order to achieve high-speed, three-dimensional locomotion. 


\section{Bibliography}

Ahmadi, M. and Buehler, M.- (1999). “-"The ARL Monopod II Running Robot: Control and Energetics, ”" in IEEE Intl. Conference on Robotics and Automation, pp. 16891694.

Berkemeier, M. (1996):- “A Model for Quadrupedal Running-in-Place in the Plane.” Proc. of the 35th FA15 950 Conference on Decision and Control. Kobe, Japan ${ }_{2}^{-}$pp. 35813586.

Berkemeier, M. (1998). “Modelling the dynamics of quadrupedal running.”- International Journal of Robotics Research. Vol. 17도. 971-985.

Brown, H. and Zeglin, G. (1998). “The bow leg hopping robot.” Proceedings of the IEEE International Conference on Robotics and Automation ${ }_{2}=$ Leuven, Belgium $_{2} \cdot$ pp. 781786.

Cepolina, F.; Moronti, M.; Sanguinet, M.; Zoppi, M.; Molfino, R. M. (2006). "Roboclimber versus landslides: design and realization of a heavy-duty robot for teleoperated consolidation of rocky walls”, IEEE Robotics and Automation Magazine, Vol. 13, No. 1, pp. 23- 31.

Furusho, J., Sano, A., Sakaguchi, M., Koizumi, E. (1995). “Realization of Bounce Gait in a Quadruped Robot with Articular-Joint- Type Legs.”. International Conference on Robotics and Automation,-: Nagoya, Japan, pp.: 697-702.

Gambaryan, P.P. (1974). “How Animals Run,”- John Wiley and Sons. New York.

Gonzalez de Santos, P., Armada, M. and Jimenez, M.A. (2000), "Ship building with ROWER”, IEEE Robotics and Automation Magazine, Vol. 7, No. 4, pp.35-43.

Herr, H.M. and McMahon, T.A. (2000). “A Trotting Horse Model.”. The International

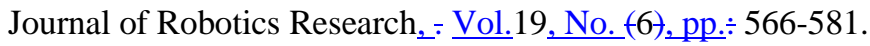

Herr, H.M. and McMahon, T.A. (2001). “A Galloping Horse Model ${ }_{2}=$-” The International Journal of Robotics Research,- Vol. 20, No. (1), pp.: 26-37.

Iida, F. and Pfeifer, R. (2004). "Cheap Rapid Locomotion of a Quadruped Robot: SelfStabilization of Bounding Gait”, In Proc. of Intelligent Autonomous Systems 8, F. Groen et al. (Eds.), IOS Press, pp. 642-649. 
Marehfka, D.W., Orin, D.E., Schmiedeler, J.P., Waldron, K.J. (2003). _Intelligent Control of Quadruped Gallops.” IEEE/ASME Transactions on Mechatronics--, No. 8, pp. :446456.

McMillan, S., Orin, D.E. and McGhee, R.B. (1996). “A computational framework for simulation of underwater robotic vehicle systems.” Autonomous Systems, $=$ No. 3, pp. $\div 253-268$.

Neishtadt, A., and Li, Z. (1991). "Stability proof of Raibert's four--legged hopper in bounding gait,:- Technical Report 578. New York state University.

Nichol J.G., Singh S.P., Waldron K.J., Palmer, L.R., and Orin, D.E. (2004). “System design of a quadrupedal galloping machine,:- International Journal of Robotics Research $_{2}=$ Vol. 23, pp. $\div 1013-1027$.

Plustech Oy Corporation. (1995). Tampere, Finland, Technical brochure.

Poulakakis, I., Papadopoulos, E. and Buehler, M. (2003). "On the Stable Passive Dynamics of Quadrupedal Running,’- Proceedings of the IEEE International Conference on Robotics and Automation,- Taipei, Taiwan ${ }_{2}=$ pp. 1368-1373.

Pugh, D.R., Ribble, E.A., Vohnout, V.Y., Bihari, T.E., Walliser, T.M., Patterson, M.R., Waldron, K.J._-(1990;_. “Technical Description of the Adaptive Suspension Vehicle”, The International Journal of Robotics Research, Vol. 9, No. 2, pp. 24-42.

Raibert, M.H. (1990). “Trotting, pacing and bounding by a quadruped robot,”: Journal of biomechanics, - No. 23, pp. $\div$ 79-98.

Raibert, M.H. (1986). “Legged Robots that balance,” MIT Press, Cambridge MA.

J. P. Schmiedeler. (2001). “The mechanics of and robotic design for quadrupedal galloping. PhD thesis,” The Ohio State University, Columbus, Ohio.

Singh, S., Waldron, K. (2005). “Attitude Estimation for Dynamic Legged Locomotion Using Range and Inertial Sensors,”- In Proc. of the IEEE International Confrence on Robotics nd Automation, Baracelona, Spain.

Song S.M. and Waldron K.J.- (1989). "Machines that Walk: The Adaptive Suspension Vehicle,”, Cambridge, MA, MIT Press.

Waldron, K.J. and Nichol J.G. (2004). “Architectural Issues in Running Machines,”- Proc. 15th CISM-IFToMM Symp. on Robot Design, Dynamics and Control. Montreal, Canada. 